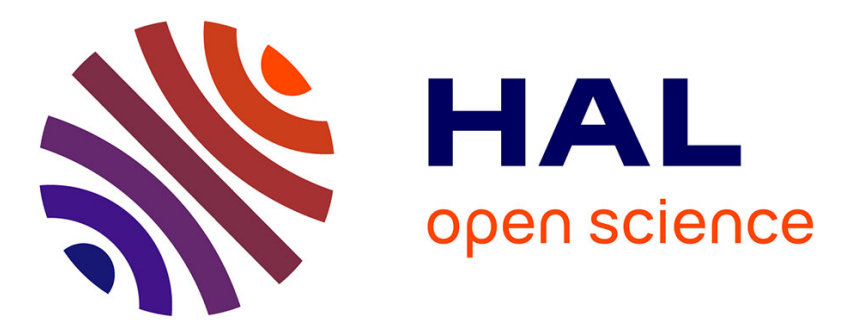

\title{
A Tool for Brain Magnetic Resonance Image Segmentation
}

Baptiste Magnier, Philippe Montesinos, Daniel Diep

\section{To cite this version:}

Baptiste Magnier, Philippe Montesinos, Daniel Diep. A Tool for Brain Magnetic Resonance Image Segmentation. 8th International Joint Conference on Computer Vision, Imaging and Computer Graphics Theory and Applications., Feb 2013, Barcelone, Spain. http://www.visapp.visigrapp.org/?y=2013. hal-00807999

\section{HAL Id: hal-00807999 \\ https://hal.science/hal-00807999}

Submitted on 4 Apr 2013

HAL is a multi-disciplinary open access archive for the deposit and dissemination of scientific research documents, whether they are published or not. The documents may come from teaching and research institutions in France or abroad, or from public or private research centers.
L'archive ouverte pluridisciplinaire HAL, est destinée au dépôt et à la diffusion de documents scientifiques de niveau recherche, publiés ou non, émanant des établissements d'enseignement et de recherche français ou étrangers, des laboratoires publics ou privés. 


\title{
A Tool for Brain Magnetic Resonance Image Segmentation
}

\author{
Baptiste Magnier ${ }^{1}$, Philippe Montesinos ${ }^{1}$, and Daniel Diep ${ }^{1}$ \\ ${ }^{1}$ LGi2P de l'Ecole des Mines d'Alès, Parc scientifique G. Besse, 30035 Nîmes cedex 1 \\ \{baptiste.magnier,philippe.montesinos,daniel.diep\}@mines-ales.fr
}

Keywords: Brain MRI regularization,edge detection, rotating filters, anisotropic diffusion.

\begin{abstract}
This paper is dedicated to a brain magnetic resonance images regularization method, preserving grey/white matter edges using rotating smoothing filters. After a preprocessing, the originality of this approach resides in the mixing of ideas coming both from pixel classification which determines roughly if a pixel belongs to a homogenous region or an edge and an anisotropic edge detector which computes two precise diffusion directions. These directions are used by an anisotropic diffusion scheme which is accurately controlled near edges and corners. Comparing our results with existing algorithms allows us to validate the robustness of our method.
\end{abstract}

\section{Introduction}

Image segmentation is a key task in many medical applications. Brain Magnetic Resonance Images (MRI) segmentation is quite complicated and challenging (Klauschen et al., 2009). Its accurate segmentation is very important for detecting tumors, edema, necrotic tissues or various diseases (Alzeimer, hydrocephaly...). A quantity of methods for automatic and semi-automatic image segmentation exists, most of them fail because of unknown noise, poor contrast, artifacts due to movements and weak boundaries. Brain MRI regularization must make possible to largely improve the diagnosis or the quantification of neural defects and diseases. However, in most of these images, the noise is an obstacle of the regularization.

In this paper, we propose a method removing the noise enhancing edges between the grey and the white matter. After a transformation of the pixel intensities distribution, we apply a method consisting in the regularization of the image according to a classification of white/grey matter edges. We consider a diffusion process driven by a partial differential equation (PDE) (Aubert and Kornprobst, 2006). In cases of homogeneous region, the purpose is to diffuse isotropically removing the noise from the image. Otherwise, in case of a contour region, we apply an anisotropic diffusion. Unlike several other diffusion scheme (Alvarez et al., 1992) (Weickert, 1999) (Tschumperlé, 2006), our control function does not solely depend on the image gradient but instead on a pre-established classification map of the initial image. Moreover in (Alvarez et al., 1992) (Weickert, 1999)
(Tschumperlé, 2006), only one direction is considered at edges and corner points, which has the effect of rounding small structures, especially when the parameter $\sigma$ used for the gradient estimation is large (a $\sigma$ value too large blurs also edges of grey/white matters). Consequently, these schemes behave like the $M C M$ scheme (Catté et al., 1995), e.g. which rounds corners after a certain number of iterations. Furthermore, when the anisotropic coefficient is too large for tensorial approaches (Weickert, 1999) (Tschumperlé, 2006), the diffusion of a high noise brings a fiber effect in homogeneous regions. In order to minimize this effect and accurately localize edges, contours junctions or corners, we considered the two directions issued from edges only in contours regions (Magnier et al., 2011b). This diffusion scheme is tuned both by the gradient magnitude and the angle between these two directions (Magnier et al., 2012).

\section{A Enhanced Segmentation Method}

This section presents the robust and efficient segmentation approach proposed for the effective segmentation of noisy brain MRI images. The approach is composed of three steps:

1. Gamma correction: we use a preprocessing of the images by gamma correction in order to expand the histogram in the range of intensities that characterizes the regions of interest (i.e. white and grey matter).

2. Edge and region classification: using a rotating 
smoothing filter, we provide a method to classify each pixel of an image into edge or region class.

3. Anisotropic diffusion: in order to enhance the detected features, we apply two types of diffusion in the image, depending on the class detected: within a region class, an isotropic diffusion is applied and in edge class, involving a rotating Gaussian derivative half-filter, we extract a gradient and two directions of edges, then we apply an anisotropic diffusion along these two directions.

In brain MRI images, white and grey matters are often dfficult to distinguish, in order to overcome this problem, we use gamma correction on the original image. Gamma correction is the name of a nonlinear operation which modifies pixel intensities in images. It can be used to improve the contrast in parts of an image, and compensate for human perception by modifying the allocation of bits used by the image coding (Poynton, 1996) (Magnier et al., 2011a). Gamma correction is defined as follows: $I_{\text {out }}(P)=(I(P))^{\gamma}$, with $\gamma>0$ and $I(P) \in[0,1]$, where $I(P)$ and $I_{\text {out }}(P)$ are respectively the input and output normalized pixel intensities.

$\gamma>1$ leads to a darker image whereas $\gamma<1$ leads to a brighter image. As the aim is to enhance edges between the white and the grey matter, we use $\gamma>1$, this operation enhances the limit between the white and the grey matters but enhances also the noise. However, our method is sufficiently robust to handle noisy images due to the thinness of the directional Gaussian filters (Magnier et al., 2011a) (Magnier et al., 2012).

\section{A Edge/Region Classifier Using Half Smoothing Filters}

In the edge classification phase, the whole image is filtered by a rotating half smoothing filter in the direction $\theta$ :

$G_{(\mu, \lambda)}(x, y, \theta)=C \cdot I_{\theta} * H(-y) \cdot e^{-\left(x^{2} / 2 \lambda^{2}+y^{2} / 2 \mu^{2}\right)}$

where $I_{\theta}$ corresponds to a rotated image ${ }^{1}$ of orientation $\theta, C$ is a normalization coefficient, $(x, y)$ are pixel coordinates, and $(\mu, \lambda)$ the standard deviations of the anisotropic Gaussian filter. Since we only require the causal part of this filter along $Y$ axis, we simply "cut" the smoothing kernel by the middle, in an operation that corresponds to the Heaviside function $H$.

\footnotetext{
${ }^{1}$ As explained in (Montesinos and Magnier, 2010), the image is oriented instead of the filter so as to increase algorithmic complexity and moreover allows use of a recursive Gaussian filter (Deriche, 1992).
}

Developped by (Montesinos and Magnier, 2010), this filter has proven to be easily implemented and adaptable to different application objectives, such as edge detection (Montesinos and Magnier, 2010) (Magnier et al., 2011a), texture suppression (Magnier et al., 2011b) and image restoration (Magnier et al., 2012). Applying this rotating filter in a $360^{\circ}$ scan to each point of an image leads to a point descriptor which represents the filtered intensity along all directions. The analysis of the descriptor enables to classify each point in two classes: flat areas (i.e. angular sector $>30^{\circ}$ estimated from de first derivative of $s(\theta)$ and a threshold $s_{t h}$ ) are associated to homogenous regions, whereas edges are characterized by a non-smooth descriptor.

\section{Two Edge Directions Extraction for Anisotropic Diffusion with PDE}

\subsection{Estimation of a Gradient and Two Edge Directions}

Steerable filters (Freeman and Adelson, 1991) (Jacob and Unser, 2004) or anisotropic edge detectors (Perona, 1992) perform well in detecting large linear structures. Close to corners however, the gradient magnitude decreases as the edge information under the scope of the filter decreases. Consequently, the robustness to noise becomes worse.

A simple solution to bypass this effect is to consider paths crossing each pixel in several directions. Wedge steerable filters introduced by Simoncelli and Farid (Simoncelli and Farid, 1996) are composed of asymmetric masks providing orientation of edges in different directions from a pixel. Unlike the Gaussian function, which is an optimal solution for the Canny criteria (Canny, 1986), wedge steerable filters have little effect near the center of the mask, whereas points in the distance are more weighted. The idea developed in (Montesinos and Magnier, 2010) was to "cut" the derivative (and smoothing) anisotropic Gaussian kernel in two parts: a first part along an initial direction, and a second part along a second direction. At each pixel of coordinates $(x, y)$, a derivation filter is applied to obtain a derivative information called $Q(x, y, \theta)$ :

$$
Q(x, y, \theta)=I_{\theta} * C_{1} \cdot H(-y) \cdot x \cdot e^{-\left(\frac{x^{2}}{2 \lambda^{2}}+\frac{y^{2}}{2 \mu^{2}}\right)}
$$


where $C_{1}$ represents a normalization coefficient. Thus, $Q(x, y, \theta)$ represents the slope of a line derived from a pixel in the perpendicular direction to $\theta$.

To obtain a gradient $\|\nabla I\|$ and its associated direction $\eta$ on each pixel $P$, we first compute global extrema of the function $Q(x, y, \theta)$, with $\theta_{1}$ and $\theta_{2} . \theta_{1}$ and $\theta_{2}$ define a curve crossing the pixel (an incoming and outgoing direction). Two of these global extrema can then be combined to maximize $\|\nabla I\|$, i.e. :

$$
\left\{\begin{array}{cl}
\|\nabla I\|= & \max _{\theta \in[0,360[} Q(x, y, \theta)-\min _{\theta \in[0,360[} Q(x, y, \theta) \\
\theta_{1}= & \underset{\theta \in[0,360[}{\arg \max }(Q(x, y, \theta)) \\
\theta_{2}= & \underset{\theta \in[0,360[}{\arg \min }(Q(x, y, \theta))
\end{array}\right.
$$

Once $\|\nabla I\|, \theta_{1}$ and $\theta_{2}$ have been obtained, the edges can be easily extracted by computing local maxima of $\|\nabla I\|$ in the direction of the angle $\eta=$ $\left(\theta_{1}+\theta_{2}\right) / 2$ followed by an hysteresis threshold (see (Montesinos and Magnier, 2010) for further details). In this paper, we are solely interested in the two directions $\left(\theta_{1}, \theta_{2}\right)$ and the gradient magnitude used in our diffusion scheme discussed bellow.

Finally, due to their thinness, rotating filters enable computing two precise diffusion orientations in the edge directions, even at high noise levels (Magnier et al., 2012). In (Magnier et al., 2011a), the authors have evaluated the edge detection used in this method with a strong noise level with and without gamma correction. A comparison with other approaches (Deriche, 1992) (Perona, 1992) shows the efficiency of this method.

\subsection{Anisotropic Diffusion in Two Directions of Edges}

As diagrammed in Fig 1 (b), the goal here is to diffuse only in the $\theta_{1}$ and $\theta_{2}$ directions in regions of pixels classified as edge points and isotropic elsewhere. We will begin by controlling the diffusion as a function of the gradient magnitude and then as a function of the angle between the two diffusion directions $\theta_{1}$ and $\theta_{2}$. Fig. 2 (e) display a diffused image without a control function wherein edges and small objects are lost and blurred (20 iterations of diffusion).

In order to control the diffusion through the gradient magnitude, as in (Perona and Malik, 1990) and (Alvarez et al., 1992), we use the following function $u(\|\nabla I\|)=e^{-(\|\nabla I\| / k)^{2}}$, with $k \in[0,1]$.

Using the anisotropic perceptual edge detector, we are now able to control the diffusion as a function of the angle between $\theta_{1}$ and $\theta_{2}$ (see eq. 3) which is labelled $\beta$ such that $\beta=\left(\theta_{1}-\theta_{2}\right)$.. At a pixel level, the closer $\beta$ is to 0 , the smaller the effect of diffusion. On the other hand, as $\beta$ moves closer to $180^{\circ}$, smoothing becomes more pronounced. Thus, the angular control function, similar to $u$, is defined by $v=e^{-[(180-\beta) /(180 \cdot h)]^{2}}$, with $h \in[0,1]$.

The diffusion process is now described by the following PDE :

$$
\frac{\partial I_{t}}{\partial t}=F_{A}\left(I_{0}\right) \Delta I_{t}+f_{c} \cdot\left(1-F_{A}\left(I_{0}\right)\right) \cdot \frac{\partial^{2} I_{t}}{\partial \theta_{1} \partial \theta_{2}}
$$

where $f_{c}=\frac{u\left(\left\|\nabla I_{0}\right\|\right)+v(\beta)}{2}$ represents the control function for both the gradient magnitude and the angle of edge orientations, $t$ is the diffusion time, $I_{0}$ the original image, $I_{t}$ the diffused image at time $t,\left(\theta_{1}, \theta_{2}\right)$ the two directions of the diffusion and lastly $F_{A}$ represents those regions where flat areas have been detected (see Section 3): $F_{A}=0$ in contour regions and $F_{A}=1$ in homogeneous regions (Magnier et al., 2011b) (Magnier et al., 2012).

In practice, for MRI images results, the values $k=0.5$ and $h=0.8$ (Fig. 1(a)) enable accurately controlling the diffusion along the edges and corners.

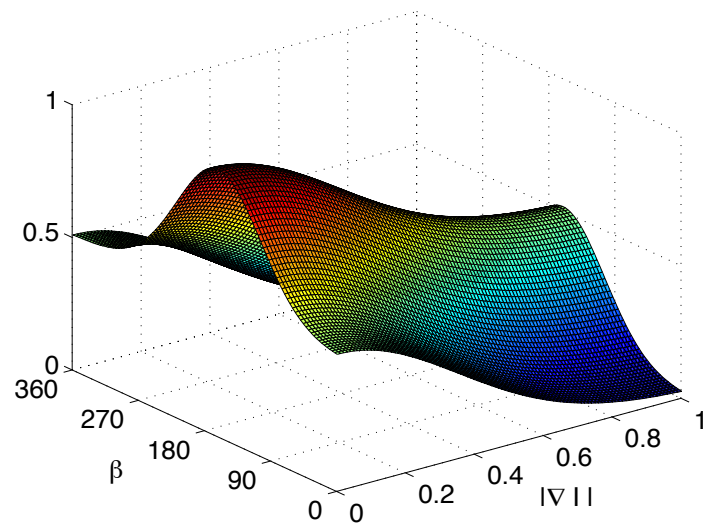

(a) Control function $f_{c}$ with $k=0.5$ and $h=0.8$

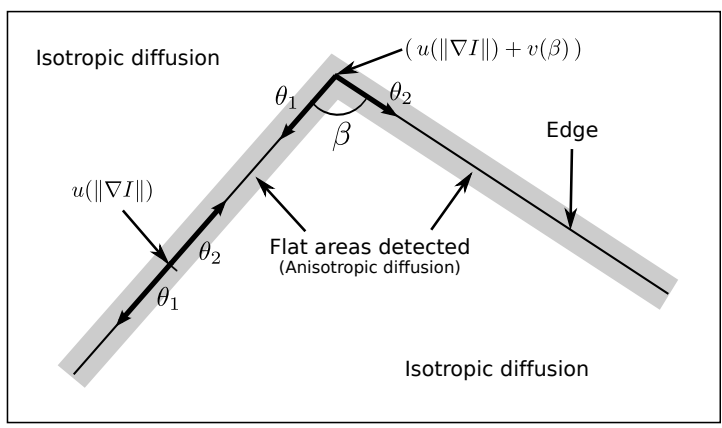

(b) Diagram of our diffusion.

Figure 1: Control functions $(u, v)$ and diagram of our diffusion scheme. 


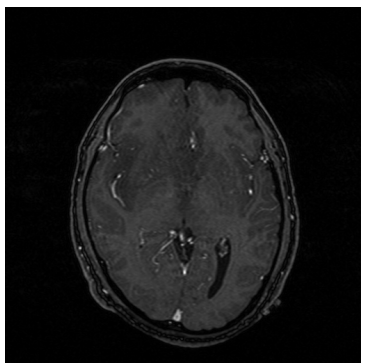

(a) Corrected image $512 \times 512$

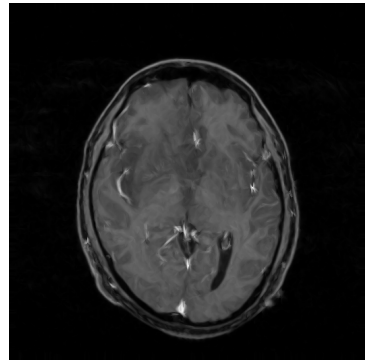

(e) Diffusion only in

$\theta_{1}$ and $\theta_{2}$ directions.

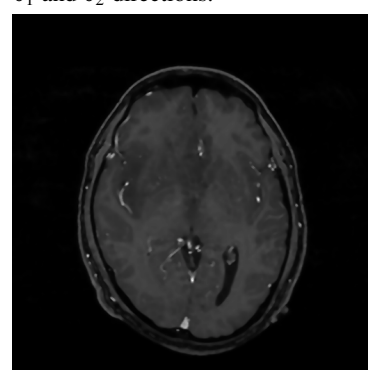

(i) Alvarez et al. (Alvarez et al., 1992), $\sigma=1,10$ iterations.

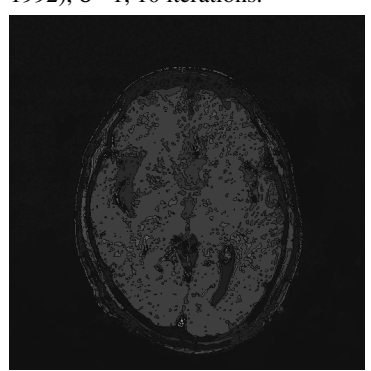

(m) Isohotes of (a)

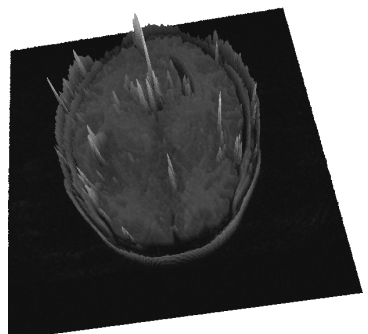

(q) Surface of (a)

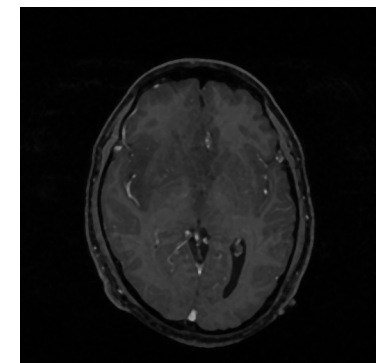

(b) Median filter $5 \times 5$

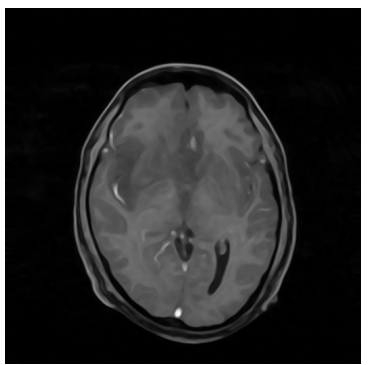

(f) MCM (Catté et al., 1995)

20 iterations.

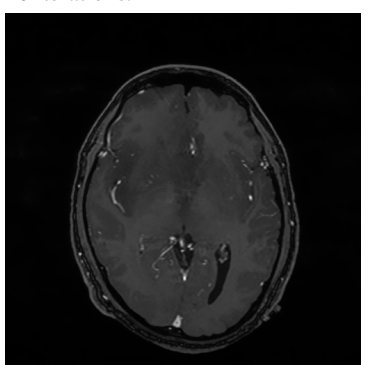

(j) Bilateral filter, variance
(Tomasi and Manduchi, 1998)

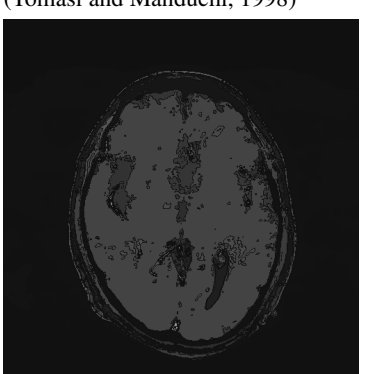

(n) Isohotes of (j)

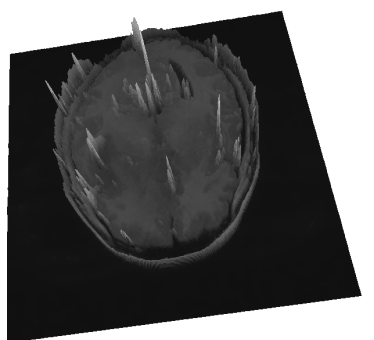

(r) Surface of (j)

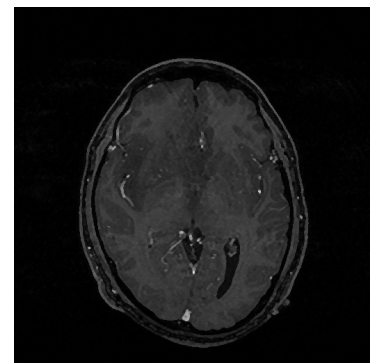

(c) Nagao filter

(Nagao and Matsuyama, 1979)

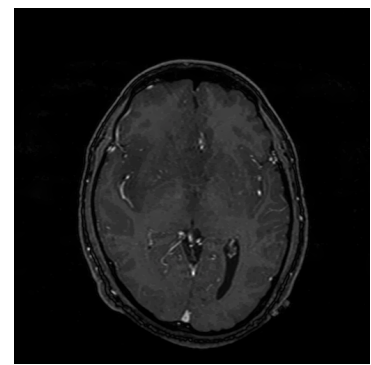

(g) PM (Perona and Malik, 1990)

100 iterations.

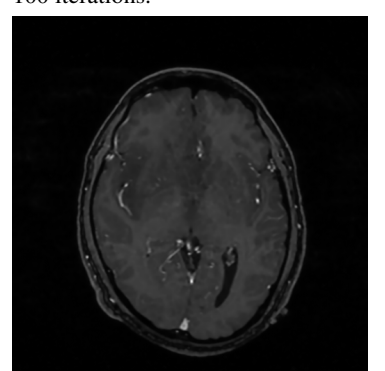

(k) Tschumperlé, $\sigma=1,10$ iterations. (Tschumperlé, 2006)

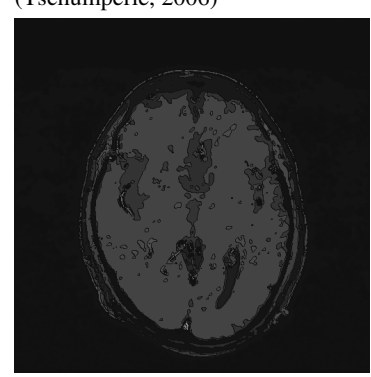

(o) Isohotes of (k)

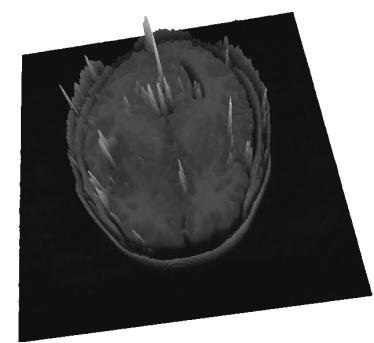

(s) Surface of (k)

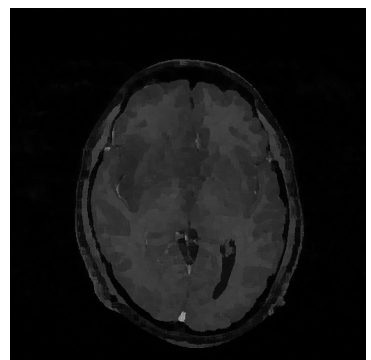

(d) Kuwahara filter

(Kuwahara et al., 1976)

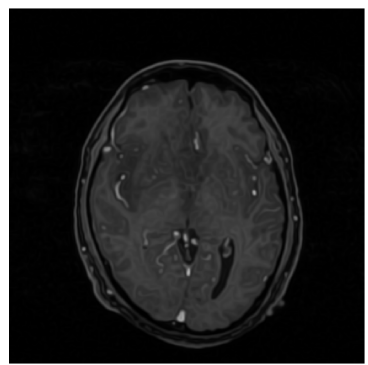

(h) Weickert, (Weickert, 1999), $\sigma=1,50$ iterations.

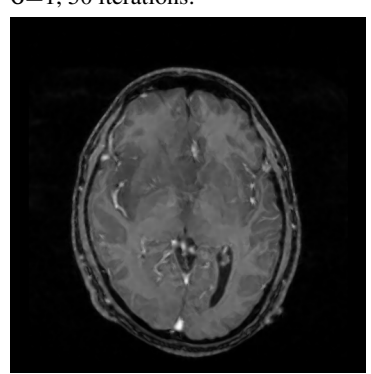

(1) Our result,

20 iterations.

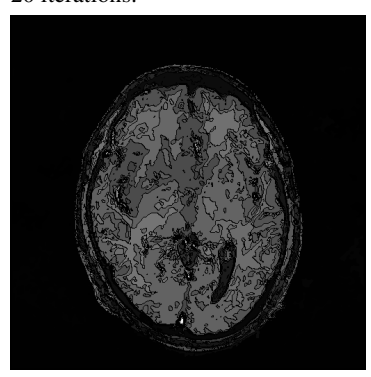

(p) Isohotes of (1)

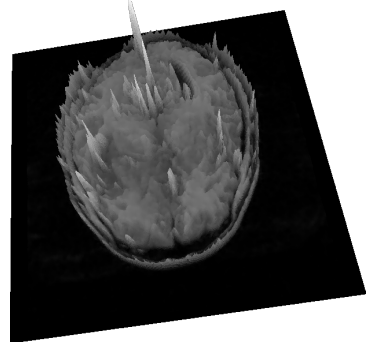

(t) Surface of (1)

Figure 2: Brain MRI restoration, isophotes and 3D elevation (normalized images). 


\section{Results and Conclusion}

In the image presented in Fig.2(a), the aim is to smooth the noise present in the images while preserving both the white and the grey matters. We used our detector with $\mu=5, \lambda=1.5$ and $\Delta \theta=5^{\circ}$ for regions classification. The threshold for the edge/region classifier $s_{t h}$ is equal to 0.002 . Parameters used in anisotropic edge detector in order to compute $\left(\theta_{1}, \theta_{2}\right)$ are $\mu=5, \lambda=1$ and $\Delta \theta=2^{\circ}$. The results of our anisotropic diffusion are presented in the Fig. 2(1). Note that the limit between the grey and the white matter is perfectly visible, Fig. 2(t) illustrates the sharpness of this edge.

We compare our result with several approaches as well as the well known median, Nagao (Nagao and Matsuyama, 1979), Kuwahara (Kuwahara et al., 1976) and bilateral filters (Tomasi and Manduchi, 1998). For these different methods, the noise is not completely removed and grey matter edges are not preserved. Tensorial approaches bring either a fiber effect to the image (Weickert, 1999) or grey matter is blurred (Tschumperlé, 2006), as for the approach of (Alvarez et al., 1992).

In order to show the efficiency of our method for noise removal and edge of grey/white matter contours enhancement, we show the image surface of our results. The 3D elevation of our result allows to see that grey/white matter edges are well preserved and sharped. Figs. $2(\mathrm{~m}),(\mathrm{n}),(\mathrm{o})$ and (p) show the isophotes (curves of the image surface of constant intensity) according to 15 levels. It is visible that our approach preserves also small objects which could be a tumor or a default inside the brain.

We have proposed in this paper a method for removing noise preserving white/grey matters edges in MRI images by pixel classification using a rotating smoothing filter followed by a PDE. Our classification method seems very promising as we have been able to classify correctly white/grey matters edges. Anisotropic diffusion in two directions provided by an edge detector using half smoothing kernels keeps edges and corners of different objects. Comparing our results with existing algorithms allows us to validate our method. Next on our agenda is to develop an internet platform where users could experiment restoration with their own images and apply a segmentation on the result.

\section{REFERENCES}

Alvarez, L., Lions, P.-L., and Morel, J.-M. (1992). Image selective smoothing and edge detection by nonlinear diffusion, ii. SIAM J. of Num. Anal., 29(3):845-866.

Aubert, G. and Kornprobst, P. (2006). Mathematical problems in image processing: partial differential equations and the calculus of variations (second edition), volume 147. Springer-Verlag.

Canny, F. (1986). A computational approach to edge detection. IEEE TPAMI, 8(6):679-698.

Catté, F., Dibos, F., and Koepfler, G. (1995). A morphological scheme for mean curvature motion and applications to anisotropic diffusion and motion of level sets. SIAM J. Numer. Anal., 32:1895-1909.

Deriche, R. (1992). Recursively implementing the gaussian and its derivatives. In ICIP, pages 263-267.

Freeman, W. T. and Adelson, E. H. (1991). The design and use of steerable filters. IEEE TPAMI, 13:891-906.

Jacob, M. and Unser, M. (2004). Design of steerable filters for feature detection using canny-like criteria. IEEE TPAMI, 26(8):1007-1019.

Klauschen, F., Goldman, A., Barra, V., Meyer-Lindenberg, A., and Lundervold, A. (2009). Evaluation of automated brain mr image segmentation and volumetry methods. Human brain mapping, 30(4):1310-1327.

Kuwahara, M., Hachimura, K., Eiho, S., and Kinoshita, M. (1976). Processing of ri-angiocardiographic images. Digital Processing of Biomedical Images, pages 187203.

Magnier, B., Montesinos, P., and Diep, D. (2011a). Fast Anisotropic Edge Detection Using Gamma Correction in Color Images. In IEEE 7th ISPA, pages 212-217.

Magnier, B., Montesinos, P., and Diep, D. (2011b). Texture Removal in Color Images by Anisotropic Diffusion. In VISAPP, pages 40-50.

Magnier, B., Montesinos, P., and Diep, D. (2012). A new region-based pde for perceptual image restoration. In VISAPP, pages 56-65.

Montesinos, P. and Magnier, B. (2010). A New Perceptual Edge Detector in Color Images. In ACIVS, volume 2, pages 209-220.

Nagao, M. and Matsuyama, T. (1979). Edge preserving smoothing. CGIP, 9:394-407.

Perona, P. (1992). Steerable-scalable kernels for edge detection and junction analysis. IMAVIS, 10(10):663-672.

Perona, P. and Malik, J. (1990). Scale-space and edge detection using anisotropic diffusion. IEEE TPAMI, 12:629-639.

Poynton, C. (1996). A technical introduction to digital video. John Wiley \& Sons, Inc. New York, NY, USA.

Simoncelli, E. and Farid, H. (1996). Steerable wedge filters for local orientation analysis. IEEE TIP, 5(9):13771382.

Tomasi, C. and Manduchi, R. (1998). Bilateral filtering for gray and color images. In International Conference on Computer Vision, pages 839-846. IEEE.

Tschumperlé, D. (2006). Fast anisotropic smoothing of multi-valued images using curvature-preserving PDE's. IJCV, 68(1):65-82.

Weickert, J. (1999). Coherence-enhancing diffusion filtering. IJCV, 31(2):111-127. 